\title{
Moving from transplant as a treatment to transplant as a cure
}

\author{
Sam Kant ${ }^{1}$ and Daniel C. Brennan ${ }^{1,2}$ \\ ${ }^{1}$ Nephrology Division and ${ }^{2}$ Comprehensive Transplant Center, Johns Hopkins School of Medicine, Baltimore, Maryland, USA.
}

\begin{abstract}
Immunosuppression continues to be a necessary component of transplantation, despite its association with a multitude of adverse effects. Numerous efforts have been made to circumvent the need for immunosuppression by using various techniques to achieve donor hyporesponsiveness. In this issue of the $J C l$, Morath et al. take this endeavor forward. Prior to transplantation, the researchers infused recipients with donor-modified immune cells and achieved immunologic hyporesponsiveness. This successful phase I trial also provides a possible avenue for achieving transplantation without the requisite immunosuppression.
\end{abstract}

\section{Immunosuppression is costly}

Solid organ transplantation is a treatment and not a cure. Its success depends on immunosuppression, which in turn has toxicity, limited efficacy, high cost, and problems with adherence. Immunosuppression is associated with an increased risk of hypertension, cardiovascular disease, diabetes, nephrotoxicity, and neurotoxicity, as well as common side effects including tremors, nausea, vomiting, and diarrhea. Immunosuppression is costly, averaging over $\$ 10,000$ per year. Nonadherence is common and a major cause for transplant failure, especially in the first year after transplantation (1). Many patients are nonadherent because of cost or toxicity, but even for adherent patients, more than half develop chronic rejection (2), and 10\%-20\% develop recurrent disease (3), with both complications potentially leading to graft loss. Thus, current immunosuppression regimes are flawed, and as a result, our endeavors to make kidney transplantation safer and more convenient and prolong graft and patient survival have stalled.

\section{Generation of tolerance}

The holy grail of successful transplantation would be a strategy devoid of immunosuppression. To give this a historical context, freemartin cattle, circa 1945 (Figure 1), were serendipitously found to have similar blood phenotypes in genetically dissimilar twins, which was explained by a common occurrence of vascular anastomosis (vessel fusion) between bovine twin embryos (4). Six years later, Medawar alluded to the phenomenon of microchimerism, the mother-to-offspring cell transfer involved in the generation of tolerance (5). In 1954, the first attempt at transplantation without immunosuppression was done between identical twin siblings; however, the recipient succumbed to recurrence of glomerulonephritis, and the graft failed after eight years (6). Despite the graft loss from recurrent disease, this first truly successful kidney transplantation paved the way for understanding the phenomenon of immunologic tolerance. The impetus for further understanding of tolerance was provided by infusions of donor bone marrow cells (DBMCs) in canine renal allograft recip-

\section{Related Article: p. 2364}

Conflict of interest: DCB receives consulting and speaker fees from CareDx and Sanofi and royalties from UpToDate. CareDx has provided research support to the Johns Hopkins School of Medicine.

Copyright: () 2020, American Society for Clinical Investigation.

Reference information: / Clin Invest. 2020;130(5):2189-2191. https://doi.org/10.1172/JCI136475.

ients (7). Starzl provided the first definitive evidence for the presence of microchimerism in bone marrow-derived cells in allograft recipients, showing ceased immunosuppression for a prolonged period without loss of the allograft (8). Since then, most tolerance-inducing treatments have and continue to use combined bone marrow or hematopoietic stem cell infusion with kidney transplantation to produce some level of chimerism that allows for the avoidance of immunosuppression (9-12). However, cytotoxic conditioning regimens, total lymphoid irradiation, or biologic agents put the patient at risk for infection and graft-versus-host disease (GVHD) and have limited applicability, since they require living donors with close HLA matching. Moreover, donor chimerism can be transient and therefore unpredictable in preventing rejection (13).

\section{Modified immune cells}

In this issue of the JCI, Morath et al. (14) sought to circumvent the GVHD issues discussed above. The authors developed a cell therapeutic called modified immune cells (MICs), which are leukapheresis-derived donor monocytes treated with mitomycin C. The resultant cells developed features of immature DCs and resulted in profound suppression of the $\mathrm{T}$ cell response. Before living donor kidney transplantation, MICs were infused into 10 patients ( 3 groups of patients received MICs at different time periods), who were subsequently followed for 1 year.

The Morath et al. study succeeded in its primary objective of demonstrating safety, as the infusions were not associated with any significant adverse effects, and no patient developed chimerism despite receiving a high volume of allogenic donor mononuclear cells (up to $1.3 \times 10^{10}$ ) before transplantation. No GVHD occurred, because the MICs did not proliferate after treatment with mitomycin C. Additionally, no de novo donor-specific antibodies (DSAs) or episodes of rejection were detect- 


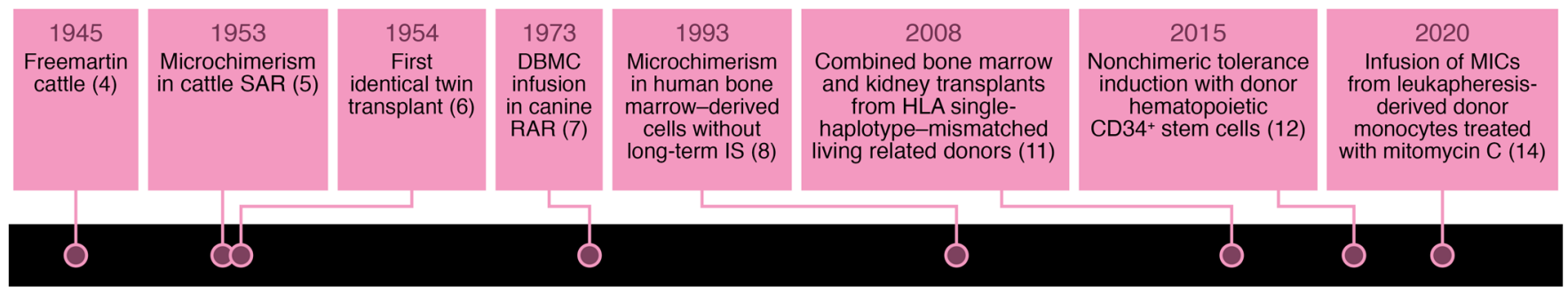

Chimerism

Figure 1. A brief history of time: tolerance from discovery to application. Timeline detailing the evolution of understanding immunologic tolerance and subsequent efforts to achieve it. RAR, renal allograft recipient; SAR, skin allograft recipient.

ed during the one-year follow-up period. Immunologic donor-specific hyporesponsiveness was achieved along with retention of responsiveness to third-party cells, demonstrating that the immune response could be redirected toward donor-specific hyporesponsiveness in the absence of chimerism. Importantly, however, some episodes of infection triggered transient donor-specific responsiveness that abated upon resolution of the infection. This transient donor-specific responsiveness may be beneficial, because none of the patients developed BK viremia (BKV) or CMV viremia, which are common donor-derived opportunistic infections that occur during the first year after transplantation. The group of patients who showed the most profound hyporesponsiveness were also off steroids at the one-year follow-up point. Also, no patients developed leukopenia, post-transplantation lymphoproliferative disorder, or diabetes. The study by Morath and colleagues provided mechanisms that explain the hyporesponsiveness to donors by demonstrating a substantial increase in $\mathrm{CD} 19^{+} \mathrm{CD} 24^{\mathrm{hi}} \mathrm{CD} 38^{\text {hi }}$ transitional B lymphocyte regulatory cells (Bregs) after transplantation. This subset of cells is instrumental in maintaining long-term allograft immunologic tolerance (15-17). Bregs exert this suppressive action through IL-10 and TGF- $\beta 1$. Serum levels of both were increased in patients treated with MICs. Most important, MIC-treated patients displayed Immune Tolerance Network (ITN) operational tolerance signatures, such as immunoglobulin kappa variable 4-1 (IGKV4-1) and immunoglobulin variable 1D-13 (IGKV1D-13). These favorable gene expression patterns are also associated with improved renal allograft function (18). It is unlikely that the results were due to maintenance immunosuppression, because a retrospective review of a non-MIC-treated control group who were on similar immunosuppression with cyclosporine or tacrolimus showed that these patients failed to develop the tolerogenic signals and profiles.

It is important to remember that Morath et al. carried out a phase I study designed to test safety but not to assess the efficacy of the treatment. Thus, only four patients underwent extensive evaluations to elucidate the mechanisms of immunologic tolerance, and the control group was selected retrospectively and included patients on tacrolimus as well as cyclosporine. Indeed, the use of cyclosporine as the calcineurin inhibitor (CNI) in the study population makes the findings less applicable to a real-world scenario in which tacrolimus would be more commonly used. It is important to note that patients who demonstrated immunologic hyporesponsiveness were kept on cyclosporine throughout the one-year follow-up period. However, the authors make a case by citing that MIC-treated animals showed significantly higher Breg $\left(\mathrm{CD} 19^{+} \mathrm{CD} 5^{+} \mathrm{CD} 1 \mathrm{~d}^{\text {hi }}\right)$ frequencies than did untreated controls in the absence of any immunosuppressive therapy, thereby ruling out a role for immunosuppression in the generation of immunologic hyporesponsiveness. In this study, patients were well HLA matched, but not as well matched as subjects in other tolerogenic protocols, with the majority having three or fewer HLA mismatches. Also, all patients were recipients of living donor transplants. The baseline characteristics of the patients assigned to groups with various MIC doses and timing of administration also differed.

\section{Future trials}

The next phase II study should address the timing and dose of the MICs. The data presented show that administration of 1.5 $\times 10^{8}$ MICs per kg BW seven days before transplantation was associated with the strongest donor-specific hyporesponsiveness as well as development of Bregs and a tolerance signature. The investigators previously used MICs generated from donor monocytes to treat a patient who experienced therapy-resistant rejection of a haploidentical stem cell transplant (19). This suggests that delivering an MIC infusion after transplantation and, intriguingly, that using MICs from deceased donors at the time of transplantation or even later could induce donor-specific hyporesponsiveness and tolerance.

There are further questions that future trials should address. Since none of the patients developed BKV in the first year of follow-up, it remains to be seen whether development of BKV would break the donor hyporesponsiveness, as BKV occurs in $15 \%$ or more of transplant recipients and is associated with the formation of de novo donor-specific antibodies (20). Also, all of the recipients were seropositive for EBV. EBV seronegativity is associated with an increased risk of post-transplantation lymphoproliferative disorders.

In conclusion, the phase I study by Morath et al. demonstrates the safety of MIC infusion and provides supportive mechanistic data for induction of donor-specific hyporesponsiveness that challenge the concept that chimerism is needed for tolerance. These preliminary findings are encouraging and warrant proceeding with phase II and III trials. If this treatment does eventually show contin- 
ued safety and efficacy, we will be witness to the realization of what transplantation originally set out to achieve: to move from a treatment to a cure.

Address correspondence to: Daniel C. Brennan, Comprehensive Transplant Center, Johns Hopkins Medical Institutions, 668A Carnegie, 600 North Wolfe Street, Baltimore, Maryland 21287, USA. Phone: 410. 955.5268; Email: dbrenna4@jhmi.edu.

1. Butler JA, Roderick P, Mullee M, Mason JC, Peveler RC. Frequency and impact of nonadherence to immunosuppressants after renal transplantation: a systematic review. Transplantation. 2004;77(5):769-776.

2. Joosten SA, Sijpkens YW, van Kooten C, Paul LC. Chronic renal allograft rejection: pathophysiologic considerations. Kidney Int. 2005;68(1):1-13.

3. Golgert WA, Appel GB, Hariharan S. Recurrent glomerulonephritis after renal transplantation: an unsolved problem. Clin JAm Soc Nephrol. 2008;3(3):800-807.

4. Owen RD. Immunogenetic consequences of vascular anastomoses between twins. Science. 1945;102(2651):400-401.
5. Anderson D, Billingham RE, Lampkin GH, Medawar PB. The use of skin grafting to distinguish between monozygotic and dizygotic twins in cattle. Heredity. 1951;5(3):379-397.

6. Krishnan N, Buchanan PM, Dzebisashvili N, Xiao H, Schnitzler MA, Brennan DC. Monozygotic transplantation: concerns and opportunities. Am J Transplant. 2008;8(11):2343-2351.

7. Caridis DT, Liegeois A, Barrett I, Monaco AP. Enhanced survival of canine renal allografts of ALS-treated dogs given bone marrow. Transplant Proc. 1973;5(1):671-674.

8. Starzl TE, et al. Chimerism and donor-specific nonreactivity 27 to 29 years after kidney allotransplantation. Transplantation. 1993;55(6):1272-1277.

9. Sasaki H, et al. Preclinical and clinical studies for transplant tolerance via the mixed chimerism approach. Hum Immunol. 2018;79(5):258-265.

10. Lowsky R, Strober S. Combined kidney and hematopoeitic cell transplantation to induce mixed chimerism and tolerance. Bone Marrow Transplant. 2019;54(Suppl 2):793-797.

11. Kawai T, et al. HLA-mismatched renal transplantation without maintenance immunosuppression. N Engl J Med. 2008;358(4):353-361.

12. Leventhal JR, et al. Nonchimeric HLA-identical renal transplant tolerance: regulatory immunophenotypic/genomic biomarkers. Am J Trans- plant. 2016;16(1):221-234.

13. Leventhal JR, Mathew JM. Outstanding questions in transplantation: Tolerance. Am J Transplant. 2020;20(2):348-354.

14. Morath C, et al. Phase I trial of donor-derived modified immune cell infusion in kidney transplantation. JClin Invest. 2020;130(5):2364-2376.

15. Shabir S, et al. Transitional B lymphocytes are associated with protection from kidney allograft rejection: a prospective study. Am J Transplant. 2015;15(5):1384-1391.

16. Chesneau M, et al. Unique B cell differentiation profile in tolerant kidney transplant patients. Am J Transplant. 2014;14(1):144-155.

17. Newell KA, et al. Identification of a B cell signature associated with renal transplant tolerance in humans. J Clin Invest. 2010;120(6):1836-1847.

18. Newell KA, Adams AB, Turka LA. Biomarkers of operational tolerance following kidney transplantation - The immune tolerance network studies of spontaneously tolerant kidney transplant recipients. Hum Immunol. 2018;79(5):380-387.

19. Kleist C, et al. Generation of suppressive blood cells for control of allograft rejection. Clin Sci. 2015;128(9):593-607.

20. Sawinski D, Trofe-Clark J. BKV viremia and development of de novo DSA in renal transplant recipients. Clin Transpl. 2015;31:249-256. 